\title{
Plasma Fibrinogen Levels As A Prognostic Biomarker In Acute Ischemic Stroke.
}

\author{
Dr. Ramiz Mahmoud Elmowafy ${ }^{1}$ Dr. Abo Zaid Abd-Allah Khodair ${ }^{2}$ Dr. Abd Elnaser Ali \\ Morad $^{3}$ Dr. Shaimaa Mohamed Kassem ${ }^{4}$. \\ Mataria Teaching Hospital, Cairo , Egypt
}

\begin{abstract}
Background: Stroke patients have significantly higher fibrinogen levels. Previous studies revealed that, hyperfibrinogenemia at the beginning of acute ischemic stroke is associated with increased risk of death within one year after stroke. Little is known about the influence of fibrinogen levels on functional outcome.

Aim: Investigate the prognostic influence of the change in the plasma fibrinogen levels after acute ischemic stroke on the functional outcome of the patients.

Methods: Fibrinogen levels were determined within 24 hours and at day 5 of stroke onset. Modified Rankin Scale (mRS) at 90 days quantified functional outcomes. Pearson's product correlation coefficient " $r$ " was used to assess linear correlation between fibrinogen levels and $m R S$.

Results Plasma fibrinogen levels are high in patients with acute ischemic stroke. The mean plasma fibrinogen level at the first 24 hours was $3.66 \pm 0.796 \mathrm{~g} / \mathrm{L}$, while after 5 days was higher with a mean of $4.72 \pm 1.669 \mathrm{~g} / \mathrm{L}$, with significant correlation with functional outcome at 90 days $p<0.0001$.

Conclusion: The present study found that not only the high fibrinogen level at onset of acute ischemic stroke was correlated with poor function outcome but also the sustained increase of the plasma fibrinogen level after 5 days of ischemic stroke.

Keywords: stroke, fibrinogen, functional outcome.

List of Abbreviations: Modified Rankin Scale (mRS).
\end{abstract}

\section{Introduction}

Fibrinogen is a coagulation factor, a major determinant of plasma viscosity and key factor for platelet activation [1]. In addition to hypertension and diabetes, fibrinogen independently predicts future ischemic stroke risk [2]. Fibrinogen also acts as an acute-phase protein and increases after stroke [3]. Clinical studies have demonstrated that increased level of inflammatory parameters related to acute phase reaction predicts unfavorable outcome in ischemic stroke patients [4]. Higher levels of plasma fibrinogen in acute ischemic stroke are associated with increased risk of unfavourable functional outcome [5], short-term mortality. [6]

\section{Patients and Methods}

The current prospective study was conducted between March 2016 and June 2016 on 30 patients with acute ischemic stroke who were admitted to Mataria teaching hospital stroke unit within 24 hours of the onset of the symptoms and whose lesion was confined according to neurological examination and computed tomography (CT brain). The exclusion criteria were: age less than 20 years old and older than 70 years old, National Institute of Health Stroke Scale (NIHSS) of the patients $>20$, recent clinical infection, concurrent major renal, hepatic and cancer disease, patient is on anticoagulant therapy at the time of onset of stroke, had surgery or major trauma in the previous month. No patient was treated with intravenous thrombolysis. All patients underwent head CT scan within 24 hours and after 72 hours of stroke onset.

Stroke severity on admission was assessed using National Institute of Health Stroke Scale (NIHSS) and modified Rankin Scale $(m R S)$. Arterial hypertension was diagnosed when there was a history of hypertension or when at least two readings of blood pressure were $\geq 140 / 90 \mathrm{mmHg}$. The diagnosis of diabetes mellitus was made when there was a history of diabetes and/or determined during admission according to the criteria of American Diabetes Association [7]. The diagnosis of dyslipidemia was made when there was history of hypercholesterolemia and/or fasting total cholesterol level $>200 \mathrm{mg} / \mathrm{dL}$, history of hypertriglyceridemia and/or fasting triglycerides level $>180 \mathrm{mg} / \mathrm{dL}$. Plasma fibrinogen level was determined on days 1 and 5 using modified Clauss method (Dade Behring, Marburg, Germany). Hyperfibrinogenemia was defined as plasma concentration $>3.5 \mathrm{~g} / \mathrm{L}$. The functional outcome at day 90 was determined using NIHSS and mRS. The favorable outcome was defined as mRS $0-1$. The study protocol was approved by local ethical committee, and fully written informed consent from all patients included or their legal representative was obtained. Categorical data were presented as number and percentages while quantitative data were expressed as mean and standard deviation, 95\% CI, median and percentiles. Pearson's product correlation coefficient " $r$ " was used to assess linear correlation between Fibrinogen levels and mRS, Values of less than 0.05 were considerate to indicate statistical significance. IBM SPSS statistics (V. 21.0, IBM Corp., USA, 2012) was used for data analysis.

\section{Results}

Thirty patients fulfilled the criteria. They were nineteen male (63.3\%) and eleven female (36.7\%), their age ranged from 40 to 69 years old, with a mean \pm SD of $57.76 \pm 7.71$ years old. Plasma fibrinogen levels were determined at day 1 and day 5 of the onset of ischemic stroke as presented at Fig (1). Three main risk factors of ischemic stroke were examined, the most prevalent risk factor was hypertension 22 (73.3\%) followed by dyslipidemia 19 (63.3\%) followed by DM 13 (43.3\%). The association between the risk factors and Fibrinogen levels at day 1 and 5 are presented at Tables (1-2). Stroke severity at day 1 and 90 of ischemic stroke were determined by NIHSS are presented at Fig (2). Level of dependency and functional 
outcome was determined at day 1 and 90 of ischemic stroke using mRS are presented at Fig (3). The corrolation between fibrinogen levels at day 1 and 5 of acute ischemic stroke with the function outcome at day 90 are presented at Fig (4-5), which show high statistically significant positive Pearson correlation $(P<0.0001)$ between modified Rankin Scale (mRS) at the follow up after three months of the onset of ischemic stroke and plasma fibrinogen levels at day 1 and 5, this means that when fibrinogen level increased the score of mRS increased and tend to worse function.

\section{Discussion}

In the present study, we found that the plasma fibrinogen level in the first 24 hours and after 5 days of the onset of ischemic stroke was high and ranged from with a mean \pm SD of $3.663 \pm 0.7968 \mathrm{~g} / \mathrm{L}$ and $4.72 \pm 1.669 \mathrm{~g} / \mathrm{L}$ respectivly as Fig 1.This is agreement with previous studies which found mean baseline plasma fibrinogen levels were higher in patients experiencing any cerebrovascular event[8-9]. Also our study found that not only the high fibrinogen level at onset of acute ischemic stroke was associated with poor outcome but also the sustained increase of the plasma fibrinogen level after 5 days of the onset of ischemic stroke. This consistent with previous studies which found that the sustained increase of fibrinogen level during stroke is associated with reduced chance of favorable outcome and independent baseline fibrinogen level. The elevated level of inflammatory markers such as CRP or fibrinogen at the beginning of stroke may reflect the burden of atherosclerosis and/or the presence of concomitant risk factors (e.g., hypertension). In addition, the blood level of these markers could rise during stroke as a part of the acute phase reaction.[10]. This can explained by clots that form in the presence of high fibrinogen concentrations may be made of thinner, more tightly packed fibers that are more resistant to fibrinolysis than clots formed at a lower concentration.

Therefore, fibrinogen levels in the early post stroke period may have implications for determining the efficacy of thrombolytic treatment[11]. An additional possible explanation for a relationship between higher fibrinogen levels and poorer outcome could be the higher blood viscosity known to be associated with higher fibrinogen levels because increased viscosity could potentially compromise micro vascular blood flow in marginally perfused brain areas. Our results were in disagreement with a previous study which found no association with either univariate or multivariate analysis between stroke outcome at 1 year and fibrinogen levels obtained within 12 hours of symptom onset in a mixed group of hospitalized patients with cerebrovascular disease[12]. This difference may be due to variation in ethnicity, method of fibrinogen assay, time of fibrinogen assay after stroke symptoms, risk factors among patients selected in each study, lastly age and sex of patients.Also our results found that fibrinogen level were high among hypertensive patients and this is in agreement with previous studies which founded that there was a moderately strong positive association between fibrinogen levels and prevalent hypertension in both men and women[13]. On the other hand another study founded that fibrinogen levels were not significantly associated with either systolic or diastolic blood pressures[14].The present study also found that fibrinogen levels in both samples were not significant among diabetic and dyslipidemic patients as in table 1-2, this is agreed with previous study [15]. While disagreed with another studies which found that fibrinogen was found to be significantly higher in patients with diabetes than control [13].Plasma fibrinogen is the promising candidate for the prediction of long-term mortality in acute stroke patients. Its increase in first several days after the onset of stroke reflects an acute phase reaction related to the early inflammatory response. This response persists in some patients worsens outcome, and increases risk of recurrent vascular event[6].

\section{In conclusion,}

The present study found that not only the high fibrinogen level at onset of acute ischemic stroke was correlated with poor function outcome but also the sustained increase of the plasma fibrinogen level after 5 days of ischemic stroke.

\section{Conflict of interest}

The authors declare that they have no conflict of interest.

Financial support and sponsorship

Nil

\section{References}

[1]. Koenig, Wolfgang. Fibrin (ogen) in cardiovascular disease: an update. Thrombosis and haemostasis, 2003, 89.4: 601-609.

[2]. Chuang, Shao-Yuan, et al. Fibrinogen independently predicts the development of ischemic stroke in a Taiwanese population Cvdfacts study. Stroke, 2009, 40.5: 1578-1584.

[3]. Ernst, Edzard; RESCH, Karl Ludwig. Fibrinogen as a cardiovascular risk factor: a meta-analysis and review of the literature. Annals of Internal Medicine, 1993, 118.12: 956-963.

[4]. Dziedzic, Tomasz. Clinical significance of acute phase reaction in stroke patients. Front Biosci, 2008, 13.1: 2922-2927.

[5]. Del Zoppo, Gregory J., et al. Hyperfibrinogenemia and functional outcome from acute ischemic stroke. Stroke, 2009, 40.5: 1687-1691.

[6]. TURAJ, Wojciech, et al. Increased plasma fibrinogen predicts one-year mortality in patients with acute ischemic stroke. Journal of the neurological sciences, 2006, 246.1: 13-19.

[7]. American Diabetes Association, et al. Diagnosis and classification of diabetes mellitus. Diabetes care, 2010, 33.Supplement 1: S62-S69.

[8]. Di Napoli, Mario; PAPA, Francesca; Bocola, Vittorio. Prognostic influence of increased C-reactive protein and fibrinogen levels in ischemic stroke. Stroke, 2001, 32.1: 133-138.

[9]. TANNE, David, et al. A prospective study of plasma fibrinogen levels and the risk of stroke among participants in the bezafibrate infarction prevention study. The American journal of medicine, 2001, 111.6: 457-463.

[10]. Swarowska, Marta, et al. The Sustained Increase of Plasma Fibrinogen During Ischemic Stroke Predicts Worse Outcome Independently of Baseline Fibrinogen Level. Inflammation, 2014, 37.4: 1142-1147.

[11]. González-Conejero, Rocio, et al. Role of fibrinogen levels and factor XIII V34L polymorphism in thrombolytic therapy in stroke patients. Stroke, 2006, 37.9: 2288-2293.

[12]. LIP, G. Y. H., et al. Sequential alterations in haemorheology, endothelial dysfunction, platelet activation and thrombogenesis in relation to prognosis following acute stroke: The West Birmingham Stroke Project. Blood Coagulation \& Fibrinolysis, 2002, 13.4: 339-347. 
[13]. Kefle, d. R.; shrestha, p. Study of fibrinogen in patients with diabetes mellitus. Nepal Med Coll J, 2010, 12.1: 34-37.

[14]. FOGARI, Roberto, et al. Associations between plasma fibrinogen levels and cardiovascular risk factors in hypertensive men. Journal of cardiovascular risk, 1994, 1.4: 341-345.

[15]. MISSOV, Rossen M., et al. Plasma fibrinogen in NIDDM: the Rotterdam study. Diabetes Care, 1996, 19.2: 157-159.

Tables

Table 1: The association between the risk factors and Fibrinogen levels at day 1

\begin{tabular}{|c|c|c|c|}
\hline \multicolumn{4}{|c|}{ Fibrinogen Level Day 1 } \\
\hline Risk Factor & $\mathrm{N}[n(\%)]$ & Mean \pm SD & $P$ Value \\
\hline Hypertension & $22(73.3)$ & & \multirow{2}{*}{0.002} \\
\hline+ & $8(26.7)$ & $3.87 \pm 0.791$ & \\
\hline- & $13(43.3)$ & $3.56 \pm 0.703$ & \\
\hline Diabetes Mellitus & $17(56.7)$ & $3.66 \pm 0.851$ & \multirow{2}{*}{$>0.05$} \\
\hline+ & $19(63.3)$ & & \\
\hline- & $11(36.7)$ & $3.58 \pm 0.819$ & \multirow{2}{*}{$>0.05$} \\
\hline Dyslipidemia & & & \\
\hline+ & &
\end{tabular}

Table 2: The association between the risk factors and Fibrinogen levels at day 5

\begin{tabular}{|c|c|c|c|}
\hline \multicolumn{4}{|c|}{ Fibrinogen Level Day 5 } \\
\hline Risk Factor & $\mathrm{N}[n(\%)]$ & Mean \pm SD & $P$ Value \\
\hline Hypertension & & & \multirow{2}{*}{0.008} \\
\hline+ & $22(73.3)$ & $5.07 \pm 1.643$ & \\
\hline- & $8(26.7)$ & $3.75 \pm 1.412$ & $>0.05$ \\
\hline Diabetes Mellitus & $13(43.3)$ & $4.92 \pm 1.57$ & \\
\hline+ & $17(56.7)$ & $4.65 \pm 1.77$ & \multirow{2}{*}{$>0.05$} \\
\hline- & $19(63.3)$ & $4.68 \pm 1.668$ & \\
\hline Dyslipidemia & $11(36.7)$ & $4.77 \pm 1.751$ & \\
\hline+ & & \\
\hline
\end{tabular}

Figures:

Fig (1): Plasma fibrinogen levels during acute stroke.

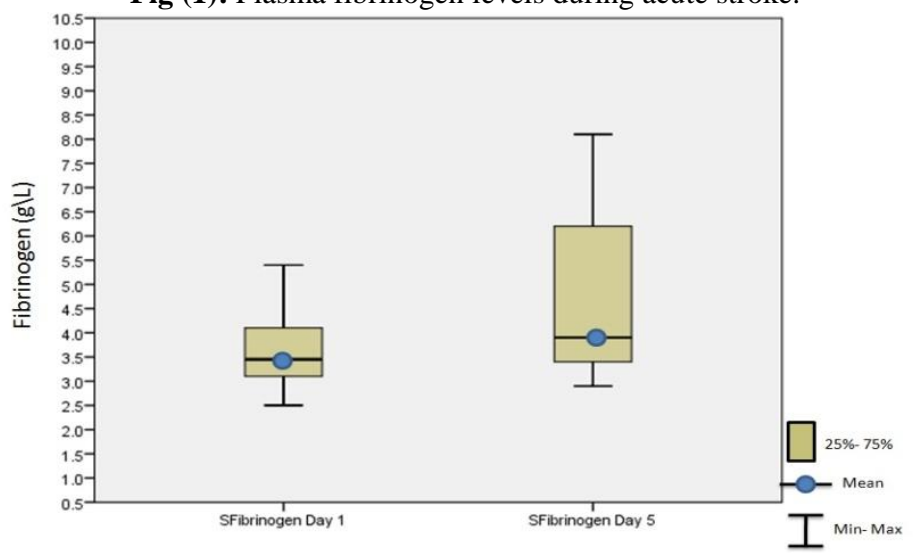

Fig (2): National Institutes of Health Stroke Scale of patients at baseline and at day 90 .

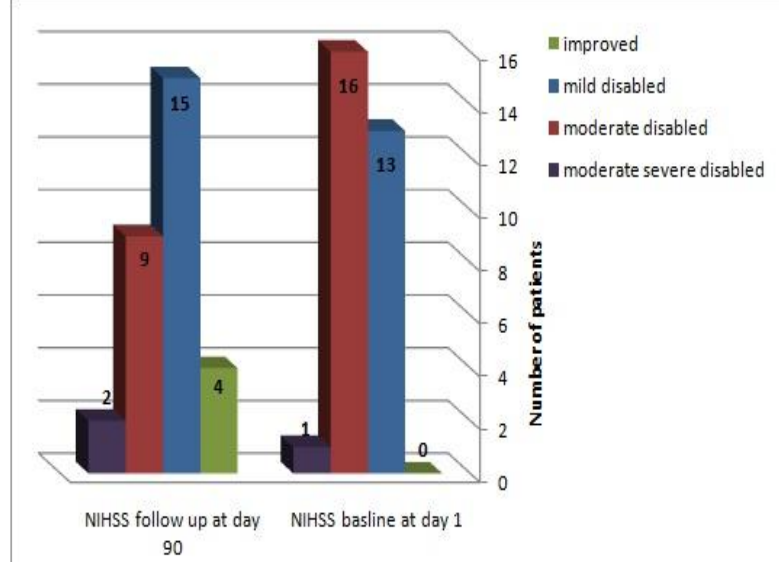

90 
Fig (3): Modified Rankin Scale of patients at baseline and at day 90.

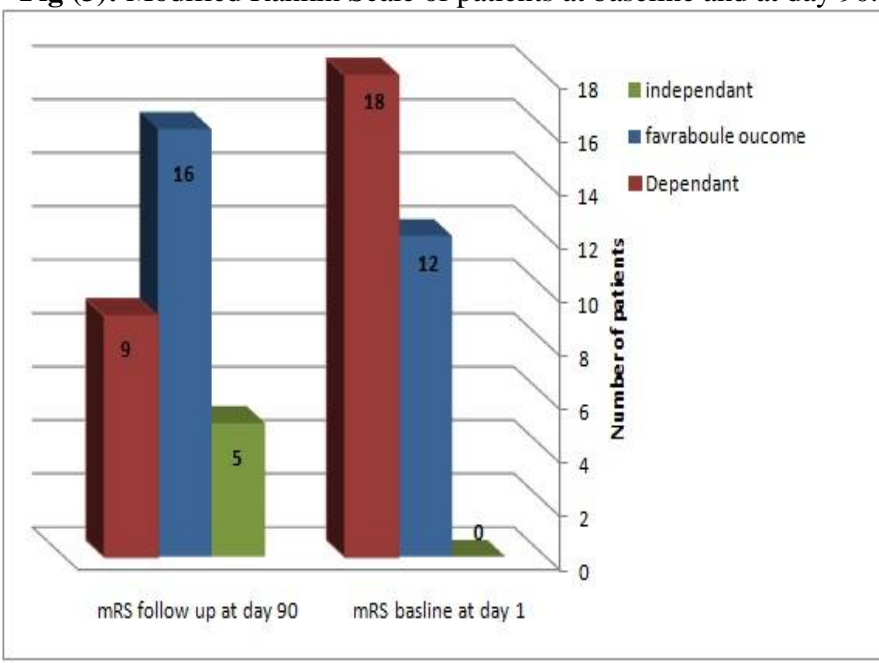

Fig (4): Scatter plot with Correlation between mRS at day 90 and day 1 plasma fibrinogen.

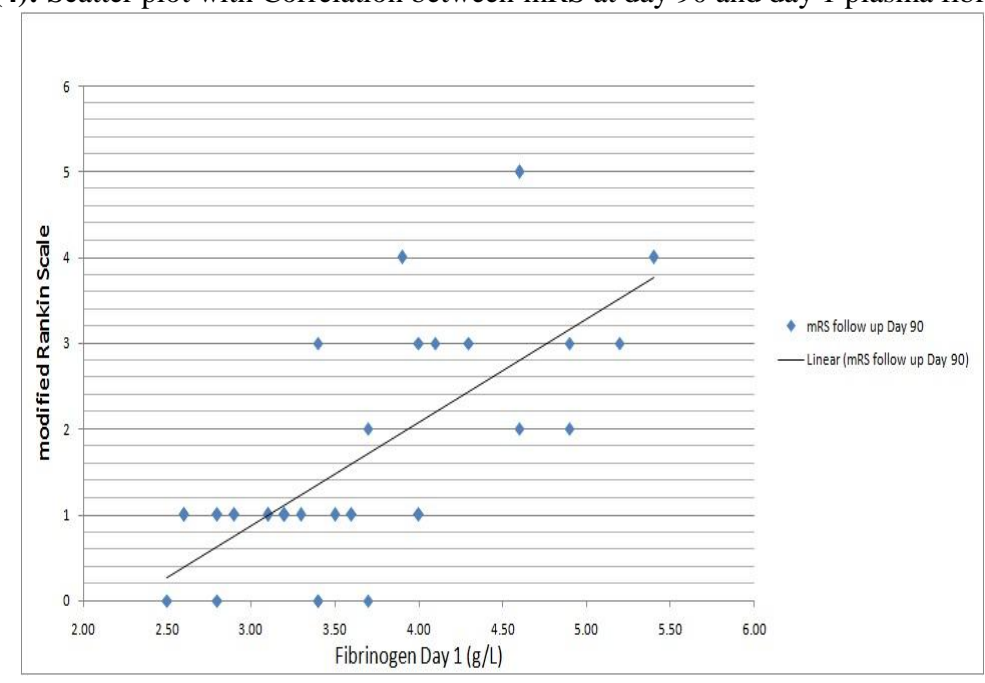

Fig (5): Scatter plot with Correlation between $\mathrm{mRS}$ at day 90 and day 5 plasma fibrinogen.

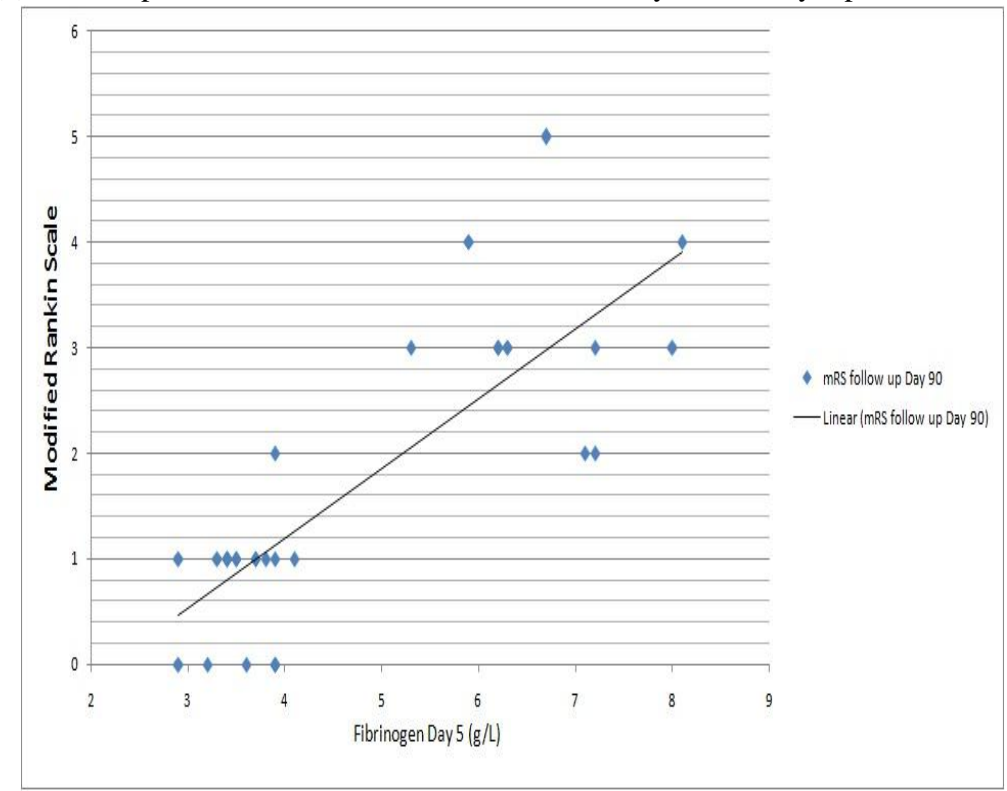

